\title{
26 Research Soure \\ Observation of short-term curative effect of femoral neck system for femoral neck fracture in adults
}

shoujin tian

soochow university affiliated zhangjiagang hospital

jianfei ge ( $\nabla$ gejianfei0271@163.com )

soochow university affiliated zhangjiagang hospital

xiaolong lin

soochow university affiliated zhangjiagang hospital

wei zhang

soochow university affiliated zhangjiagang hospital

su liu

soochow university affiliated zhangjiagang hospital

xiaolei sheng

soochow university affiliated zhangjiagang hospital

\section{Research Article}

Keywords: Femoral neck system, Femoral neck fracture, femoral neck shortening, adults

Posted Date: February 21st, 2022

DOI: https://doi.org/10.21203/rs.3.rs-1367900/v1

License: (c) (i) This work is licensed under a Creative Commons Attribution 4.0 International License.

Read Full License 


\section{Abstract \\ Purpose}

The femoral neck system is a potentially effective implant for treating femoral neck fractures, However, there are few studies on the postoperative outcomes. This study aimed to evaluate short-term curative effect of FNS for femoral neck fracture in adults.

\section{Methods}

We retrospectively collected data from 29 patients who underwent surgery treated with FNS in our hospital between June 2019 and November 2020. General data (such as age, gender, type of fracture, cause of injury) of all patients were collected, meanwhile, the operative time, intraoperative blood loss, number of fluoroscopies, time before surgery were recorded. hip function was evaluated using the Harris Hip Score (HHS) six months and one year after surgery. At last, we recorded complications, including femoral head necrosis, nonunion, and femoral neck shortening.

\section{Results}

All the patients completed at least 12 months(average 16.07 months) follow-ups, the quality of reduction were assessed: excellent in 9 patients, good in 16 patients and fair in four. The mean femoral neck shortening is $3.87 \mathrm{~mm}$,four patients achieved bone healing at two months demonstrated by radiograph. Nonunion occurred in one patient and osteonecrosis of the femoral head occurred in two.

\section{Conclusion}

In the short-term observation, satisfactory results showed in the treatment of femoral neck fractures in adults with FNS. Complications such as osteonecrosis of femoral head or nonunion were rare. Patients treated with FNS obtained good hip function recovery by early partial-weight bearing.

\section{Introduction}

Hip fractures, especially femoral neck fractures, had became a serious problem over the world and a great challenge for orthopaedic surgeon[1].Data of the International Osteoporosis Foundation (IOF) in 2012 showed that incidence of hip fracture was from $55 / 100,000$ to $439 / 100,000$ and femoral neck fracture had the highest incidence in hip fractures.

Hip arthroplasty is generally considered as an effective treatment for unstable femoral neck fractures in the elderly, meanwhile, conservative treatment or internal fixation is an appropriate option for treating stable femoral neck fractures in adults. Femoral neck fractures may cause a series of complications 
among bedridden patients such as: deep vein thrombosis, hypostatic pneumonia, pressure ulcers, urinary tract infection, etc. Patients treated with conservative treatment may also risk nonunion or osteonecrosis of the femoral head(ONFH) in the medium and long term observation. The current mainstream view is that early surgery after injury is an effective therapeutic schedule, especially in the elderly[2, 3]. Based on the stability of the fracture, early patient mobilization can be achieved postoperatively, and this procedure significantly reduce the risk of complications and mortality.

Surgical procedures for femoral neck fractures in adults include multiple cannulated screws, dynamic hip screw (hereinafter referred to as DHS), and locking plate, etc. However, unsatisfactory therapeutic effect often happens, and there are literatures showing a reoperation rate of $27 \%[4,5]$. DHS has outstanding mechanical stability, but for implanting, it needs complicated procedures, a larger incision, and a longer operation time, these defects lead to $10 \%$ infection and revision rate after surgery[6]. Cannulated screw internal fixation is minimally invasive and it costs less time of surgery, but its mechanical stability is poor, patients can not walk without crutches and with partial load in the early stage postoperatively. Moreover, screw withdrawal may occur during the stage of bone healing resulting in irritation of the lateral soft tissue of the greater trochanter, and patients may experience discomfort or even pain [7].

In recent years, a new implant named as femoral neck system (hereinafter referred to as FNS) was developed. It was designed for the treatment of the femoral neck fracture. According to the biomechanical experimental data, The bolt of FNS owns advanced angular stability and withdraw resistance which allows a maximum of $20 \mathrm{~mm}$ collapse with no protrusion, meanwhile, the screw companied with the bolt provides rotational stability.

However, few studies have described the short-term outcomes of FNS for treating femoral neck fractures in adults. The present study aimed to share the short-term outcomes of the treatments of using FNS.

\section{Materials And Methods}

\section{Inclusion and exclusion criteria}

Informed consent was obtained and signed by all included patients.

The inclusion criteria were as follows:1. Unilateral fractures of the femoral neck;2.Patient's age range from 18-65 years;3.Fresh factures(less than 7 days since injury);4.Closed fractures.

The exclusion criteria were as follows:1. Pathological femoral neck fractures;2. Patients less than 18 or more than 65 years of age;3. Ipsilateral fractures of trochanter or shaft of the femur;4.patients with poor compliance.

\section{Surgical procedures}

The patient lay on an orthopaedic traction bed after epidural or general anaesthesia. Closed reduction was performed under C-arm fluoroscopy until the I and II levels achieved, which was assessed using the 
Garden reduction index. A longitudinal incision of about $4 \mathrm{~cm}$ was made parallel to the femoral shaft under the greater trochanter. Then, a $2.5 \mathrm{~mm}$ guide pin was placed in the anterior superior part of the femoral neck to maintain the fracture reduction. A $130^{\circ}$ guide device was placed in along the femoral neck, then the central guide was place in the center of femoral neck in the anteroposterior and lateral position, $5 \mathrm{~mm}$ from the tip of the guide pin to the subchondral bone is the best position. After reaming along the central guide, the assembled bolt-and-plate was inserted into the femoral head. Subsequently, locking screw and anti-rotation screw were in turn implanted.(Figs. 1 and 2).

\section{Perioperative management}

Respective antibiotic were given 30 minutes preoperatively and in 24 hours postoperatively To reduce pain caused by surgery, celecoxib or tramadol were used. One dose of Low-molecular-weight heparin(0.2$0.4 \mathrm{ml}$,determined by weight of patient) or enoxaparin $(0.4 \mathrm{ml})$ were injected in 12 hours postoperatively and each day until discharge.

All patients were instructed to take quadriceps isometric contraction exercise and ankle pump exercise on bed on the day after surgery. Then, patients with stable fracture were permitted to walk with partial weight bearing. After discharge, rivaroxaban(10 mg per day) were given orally in 6 weeks. Patients were requested to return to the clinic for follow-up at 1,2,3,6,and 12 months postoperatively and then every 6 months subsequently.

\section{Outcome evaluation}

General data such as operation time, intraoperative fluoroscopy times, blood loss and length of incision were collected, meanwhile, postoperative complications such as infection of incision and implant failure (significantly screw withdraw, screw cutout, fixation failure and fracture displacement) were recorded. Lateral radiographs of the femoral neck and anteroposterial radiographs of the pelvis were taken on the second day after surgery, then the reduction quality of the femoral neck fracture was evaluated by the reduction criteria proposed by Haidukewych[8] as follows.: Excellent $\left(<2 \mathrm{~mm}\right.$ of displacement and $<5^{\circ}$ of angulation in any plane), good ( 2 to $5 \mathrm{~mm}$ of displacement and/or $5^{\circ}$ to $10^{\circ}$ of angulation), fair $>5$ to 10 $\mathrm{mm}$ of displacement and/or $>10^{\circ}$ to $20^{\circ}$ of angulation), or poor ( $>10 \mathrm{~mm}$ of displacement and/or $>20^{\circ}$ of angulation). The progression of bone healing, fixation failure(screw withdrawal, screw cutout, fixation failure and fracture displacement) and necrosis of femoral head were recorded according to X-ray (CTscan if necessary). Harris Hip Score was used to assess the hip function at 3 months, 6 months and 1 year after surgery, with a total score of 100 points. $90-100$ points mean excellent, 80-89 points to good, 70-79 points to fair, less than 70 is poor.

\section{Results}

Finally, a total of 29 patients, 13 male and 16 female, with an average age of 51.97 years (range 28-64 years),met the inclusion criteria from June 2019 to November 2020 and all of them completed at least 12 


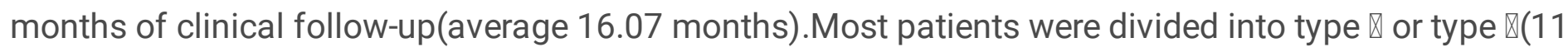
and 13, respectively) based on the Garden classification(Table 1).

\begin{tabular}{|c|c|}
\hline \multicolumn{2}{|l|}{ Table1: Statistics of patients } \\
\hline NO. of the patients/no. of the fractures & $29 / 29$ \\
\hline Gender(M/F) & $13 / 16$ \\
\hline Age(year, $\bar{x} \pm s \rrbracket$ & $52.66 \pm 11.64$ \\
\hline Side(L/R) & $17 / 12$ \\
\hline \multicolumn{2}{|l|}{ Cause of injury } \\
\hline Vehicle accident & 12 \\
\hline Fall from a standing height & 13 \\
\hline Fall from height & 4 \\
\hline \multicolumn{2}{|l|}{ Fracture type(Garden classification) } \\
\hline ૫ & 1 \\
\hline ૫ & 4 \\
\hline प & 11 \\
\hline ( & 13 \\
\hline Time before surgery(day, $\bar{x} \pm s)$ & $3.90 \pm 1.37$ \\
\hline No. of intraoperative radiograph $(\bar{x} \pm s)$ & $18.03 \pm 4.02$ \\
\hline Blood loss $(m l, \bar{x} \pm s)$ & $54.83 \pm 15.50$ \\
\hline Quality of reduction & . \\
\hline Excellent & 9 \\
\hline Good & 16 \\
\hline Fair: & 4 \\
\hline Poor & 0 \\
\hline Mean duration of follow-up (Range) & 16.07 months(12-29 months) \\
\hline
\end{tabular}


Table2: Postoperative statistics

\begin{tabular}{|c|c|c|}
\hline Osteonecrosis & \multicolumn{2}{|l|}{$2(6.9 \%)$} \\
\hline Nonunion & \multicolumn{2}{|l|}{$1(3.4 \%)$} \\
\hline Screw cutout & \multicolumn{2}{|l|}{0} \\
\hline The degree of femoral neck shortening $₫ \mathrm{~mm} \rrbracket$ & \multicolumn{2}{|l|}{$3.87 \pm 0.22$} \\
\hline$<5$ & \multicolumn{2}{|l|}{15} \\
\hline $5-10$ & \multicolumn{2}{|l|}{9} \\
\hline$>10$ & \multicolumn{2}{|l|}{0} \\
\hline Coxa vara & \multicolumn{2}{|l|}{$1(3.4 \%)$} \\
\hline Bone healing time(average) & \multicolumn{2}{|l|}{$2.86 \mathrm{M}$} \\
\hline \multicolumn{3}{|l|}{ Harris score $(\overline{\mathrm{x}} \pm s)$} \\
\hline $3 \mathrm{M}$ & \multicolumn{2}{|l|}{$70.55 \pm 3.40$} \\
\hline $6 \mathrm{M}$ & \multicolumn{2}{|l|}{$84.34 \pm 3.47$} \\
\hline $12 \mathrm{M}$ & \multicolumn{2}{|l|}{$88.55 \pm 2.29$} \\
\hline Complications according to fracture type & $\begin{array}{lll} & \square & \square\end{array}$ & $\square$ \\
\hline Osteonecrosis & $0 \quad 0$ & 2 \\
\hline Nonunion & $0 \quad 0$ & 1 \\
\hline
\end{tabular}

The average time from injury to surgery was 3.9 days(range 2-7 days), patients with displaced fractures accepted closed reduction after general or epidural anesthesia, then the quality of reduction was assessed: excellent in nine patients, good in 16 patients and fair in four(Table 2).

During the clinical follow-up, bone union was achieved in 28 patients. The average bone healing time was 2.86 months, bone nonunion occurred in one patient, two cases of ONFH were diagnosed. The patient with bone nonunion had accepted 12 months follow-up, and he refused further treatment because he had already returned to the normal life and there was only occasional slight pain in the anterior of the hip when he walked.

We measured and assessed the degree of femoral neck shortening using the XYZ-axis measurement[9] (Fig.1)in the twelfth month. Fifteen patients had $<5 \mathrm{~mm}$ femoral neck shortening, meanwhile, nine patients had $5-10 \mathrm{~mm}$ shortening, shortening more than $10 \mathrm{~mm}$ had not been observed in the end .

\section{Discussion}


For the treatment of femoral neck fracture, there are many difficulties, mainly including: 1. It needs early surgery to accelerate the reconstruction around the fracture; 2 . It needs rigid internal fixation, especially in the treatment of Pauwels type III femoral neck fracture, a rigid implant should provide stable shear resistance and rotation resistance; 3 . The prevailing view is that femoral neck shortening limits patients' physical function, including gait, hip abduction and mobility[10]. Effective methods are required to reduce the occurrence or degree of femoral neck shortening during bone healing postoperatively. Stockton [11] reported that the proportion of femoral neck shortening greater than $5 \mathrm{~mm}$ after surgery was $54 \%$ under 60 years old patients with femoral neck fracture, the proportion greater than $10 \mathrm{~mm}$ was $32 \%$; 4 . Based on the negative impact of femoral head necrosis on hipt function, measures should be taken to reduce the incidence of femoral head necrosis. DHS or multiple canulated screws own limit ability to solve the above difficulties satisfactorily. In addition, due to the development of the concept of enhanced recovery after surgery (ERAS), patients strongly require early recovery of hip function postoperatively, therefore, choosing an appropriate implant had became an important consideration. On the basis of DHS and multiple cannulated screws procedures, BDSF method(the biplane double-supported screw fixation method) [12], DHS with anti-rotation screw, buttress plate located on the anteroinferior femoral neck [13], rhomboid fixation of four cannulated screws[14], DHSA (an anti-rotation blade instead of lag screw), two parallel and one transverse cancellous lag screws strategy[15] were developed. The aim of applying BDSF method and cannulated screw rhomboid fixation was to disperse the stress load on the screws in femoral head and neck. but the anti-detachment and anti-shear properties of rhomboid fixation is significantly weaker compared with three parallel and one off-axis screws strategy[16],meanwhile, BDSF method and "two parallel and one transverse cancellous lag screws "strategy still require a further research about their biomechanical properties. Then, the buttress plate combined with cannulated screws, DHS or DHSA owns excellent biomechanical properties, but all of them require an invasive procedure with more blood loss and larger soft tissue dissection, especially in the treatment of Pauwels type III with osteoporosis, there is an increased risk of nonunion and osteonecrosis of the femoral head in the longterm clinical observation[17]. Therefore, none of the above surgical strategies simultaneously owns the characteristics of excellent biomechanical properties, shear resistance, rotation resistance and minimally invasive implantation procedures.

Through biomechanical test in cadaveric specimens, FNS showed better stiffness and shear resistance than three inverted cannulated screws in the treatment of Pauwels type III femoral neck fractures, meanwhile, similar biomechanical properties were reported between FNS, DHS-screw and DHS-blade[18]. The result indicated that FNS is reliable in the treatment of femoral neck fracture, more significantly, its plate length is shorter than DHS which means advantages of minimally invasive incision, less muscle dissection and blood loss. In addition, FNS provides sliding compression in the fracture site. Combined with the anti-rotation screw, the structure allows patient to walk with partial weight-bearing in early stage postoperatively, the hip joint therefore obtain earlier rehabilitation and the risk of complications such as deep venous thrombosis, digestive system dysfunction and hypostatic pneumonia caused by prolonged recumbency. In this study, four patients acquired bone healing demonstrated by radiograph at 2 months after surgery(average 2.73 months), 
Femoral neck shortening is an important limitation that may affect gait pattern and muscle strength. As described previously, thirty-two percent of patients(age between 18 and 60 years) had $\geq 10 \mathrm{~mm}$ of femoral neck shortening [11],in another study, thirty-three percent of patients(age between 50 and 80 years) had $\geq 15 \mathrm{~mm}$ of femoral neck shortening[19]. In geriatric patients, shortening $\geq 10 \mathrm{~mm}$ had significantly lower SF-36 physical functioning scores compared with patients with $<5 \mathrm{~mm}$ of shortening[20]. FNS theoretically has a limit of $20 \mathrm{~mm}$ in controlling the degree of postoperative femoral neck shortening, in our study, none of patients had more than $10 \mathrm{~mm}$ shortening of femoral neck. The possibility of greater shortening in the mid- and long-term observation requires further evaluation with more cases, and there are still poor clinical data for the treatment of elderly and osteoporosis.

With the development of 3D printing technology, we had tried to use three-dimensional CT date of patient to produce a personalized customized 3D printing guide plate, this plate perfectly fits the lateral wall of the inferior region of the greater trochanter with three fixed-angle channels on it, the channels were used for implanting anterior superior anti-rotation guide pin ,guide pin of bolt and distal locking screw. This guide plate is significantly helpful for reducing the time to repeatedly adjust the position of guide pins, the blood loss and the Intraoperative fluoroscopy frequency, which are beneficial for patients.

We acknowledge several shortcomings of this study. First, a larger sample size is required for improving reliability. Second, a longer follow-up period is needed to evaluate the postoperative complications of femoral neck fracture treated by FNS. Third, it will be helpful to obtain more valuable results if we design a prospective study.

\section{Conclusion}

In the treatment of adult femoral neck fracture, FNS can be implanted through a single minimally invasive incision after closed reduction, the incision length can be controlled less than $5 \mathrm{~cm}$. Compared with three inverted cannulated screws and DHS, its biomechanical properties are improved, patients with stable fracture were able to walk with partial weight bearing, and the short-term therapeutic effect is satisfactory for now.

\section{Declarations}

\section{Consent for publication}

Ethics approval and consent to participate The study was approved by the Ethical Committee of the soochow university affiliated zhangjiagang Hospital. Written informed consent was obtained from all participants.

\section{Competing interests}

The authors declare that they have no competing interests. 


\section{Founding}

Project of Jiangsu commission of health, NO.Z2021086

\section{Authors' contributions}

$\mathrm{Y}-\mathrm{Z}$ was a major contributor in writing the manuscript. W-Z was a contributor in the data collecting. SJ-T, $S-L$, and XL-S were contributors in writing the manuscript. XL-L analysed the data and draw the figures. JF-G was responsible for reviewing and editing the manuscript. The authors read and approved the final manuscript.

\section{Acknowledgements}

Not applicable.

\section{References}

1. Kanis JA, Oden A, McCloskey EV, et al. A systematic review of hip fracture incidence and probability of fracture worldwide. Osteoporos Int 2012; 23: 2239-2256. 2012/03/16. DOI: 10.1007/s00198-0121964-3.

2. Okike $\mathrm{K}$ and Hasegawa IG. Current Trends in the Evaluation and Management of Nondisplaced Femoral Neck Fractures in the Elderly. J Am Acad Orthop Surg 2021; 29: e154-e164. 2020/11/18. DOI: 10.5435/JAAOS-D-20-00349.

3. Florschutz AV, Langford JR, Haidukewych GJ, et al. Femoral neck fractures: current management. Journal of orthopaedic trauma 2015; 29: 121-129. 2015/01/31. DOI: 10.1097/BOT.0000000000000291.

4. Campenfeldt $P$, Hedstrom M, Ekstrom W, et al. Good functional outcome but not regained health related quality of life in the majority of 20-69 years old patients with femoral neck fracture treated with internal fixation: A prospective 2-year follow-up study of 182 patients. Injury 2017; 48: 27442753. DOI: 10.1016/j.injury.2017.10.028.

5. Bartels S, Gjertsen JE, Frihagen F, et al. High failure rate after internal fixation and beneficial outcome after arthroplasty in treatment of displaced femoral neck fractures in patients between 55 and 70 years. Acta orthopaedica 2018; 89: 53-58. DOI: 10.1080/17453674.2017.1376514.

6. Lin JC and Liang WM. Outcomes after fixation for undisplaced femoral neck fracture compared to hemiarthroplasty for displaced femoral neck fracture among the elderly. BMC musculoskeletal disorders 2015; 16: 199. DOI: 10.1186/s12891-015-0671-6.

7. Stiasny J, Dragan S, Kulej M, et al. Comparison analysis of the operative treatment results of the femoral neck fractures using side-plate and compression screw and cannulated $\mathrm{AO}$ screws. Ortopedia, traumatologia, rehabilitacja 2008; 10: 350-361.

8. Haidukewych GJ, Rothwell WS, Jacofsky DJ, et al. Operative treatment of femoral neck fractures in patients between the ages of fifteen and fifty years. The Journal of bone and joint surgery American 
volume 2004; 86: 1711-1716. DOI: 10.2106/00004623-200408000-00015.

9. Boraiah S, Paul O, Hammoud S, et al. Predictable healing of femoral neck fractures treated with intraoperative compression and length-stable implants. J Trauma 2010; 69: 142-147. 2009/12/17. DOI: 10.1097/TA.0b013e3181bba236.

10. Zlowodzki M, Jonsson A, Paulke R, et al. Shortening after femoral neck fracture fixation: is there a solution? Clinical orthopaedics and related research 2007; 461: 213-218. 2007/04/07. DOI: 10.1097/BLO.0b013e31805b7ec4.

11. Stockton DJ, Lefaivre KA, Deakin DE, et al. Incidence, Magnitude, and Predictors of Shortening in Young Femoral Neck Fractures. Journal of orthopaedic trauma 2015; 29: e293-298. DOI: 10.1097/BOT.0000000000000351.

12. Filipov 0 . Biplane double-supported screw fixation (F-technique): a method of screw fixation at osteoporotic fractures of the femoral neck. European journal of orthopaedic surgery \& traumatology : orthopedie traumatologie 2011; 21: 539-543. DOI: 10.1007/s00590-010-0747-9.

13. Mir $\mathrm{H}$ and Collinge $\mathrm{C}$. Application of a medial buttress plate may prevent many treatment failures seen after fixation of vertical femoral neck fractures in young adults. Medical hypotheses 2015; 84 : 429-433. DOI: 10.1016/j.mehy.2015.01.029.

14. Wang Z, Guan J, Wu M, et al. [Comparison of effectiveness of two cannulated screw configurations in the treatment of femoral neck fractures in young adults]. Zhongguo xiu fu chong jian wai ke za zhi = Zhongguo xiufu chongjian waike zazhi = Chinese journal of reparative and reconstructive surgery 2021; 35: 318-322. 2021/03/16. DOI: 10.7507/1002-1892.202009103.

15. Guimarães JAM, Rocha LR, Noronha Rocha $T H$, et al. Vertical femoral neck fractures in young adults: a closed fixation strategy using a transverse cancellous lag screw. Injury 2017; 48: S10-S16. DOI: 10.1016/s0020-1383(17)30769-6.

16. Jiang $D$, Zhan $S$, Wang $L$, et al. Biomechanical comparison of five cannulated screw fixation strategies for young vertical femoral neck fractures. J Orthop Res 2021; 39: 1669-1680. 2020/10/10. DOI: 10.1002/jor.24881.

17. Zhang YL, Chen S, Ai ZS, et al. Osteonecrosis of the femoral head, nonunion and potential risk factors in Pauwels grade-3 femoral neck fractures: A retrospective cohort study. Medicine 2016; 95: e3706. DOI: 10.1097/MD.0000000000003706.

18. Stoffel K, Zderic I, Gras F, et al. Biomechanical Evaluation of the Femoral Neck System in Unstable Pauwels III Femoral Neck Fractures: A Comparison with the Dynamic Hip Screw and Cannulated Screws. Journal of orthopaedic trauma 2017; 31: 131-137. DOI: 10.1097/BOT.0000000000000739.

19. Zielinski SM, Keijsers NL, Praet SF, et al. Femoral neck shortening after internal fixation of a femoral neck fracture. Orthopedics 2013; 36: e849-858. 2013/07/05. DOI: 10.3928/01477447-20130624-13.

20. Zlowodzki M, Brink O, Switzer J, et al. The effect of shortening and varus collapse of the femoral neck on function after fixation of intracapsular fracture of the hip: a multi-centre cohort study. $J$ Bone Joint Surg Br 2008; 90: 1487-1494. 2008/11/04. DOI: 10.1302/0301-620X.90B11.20582. 


\section{Figures}

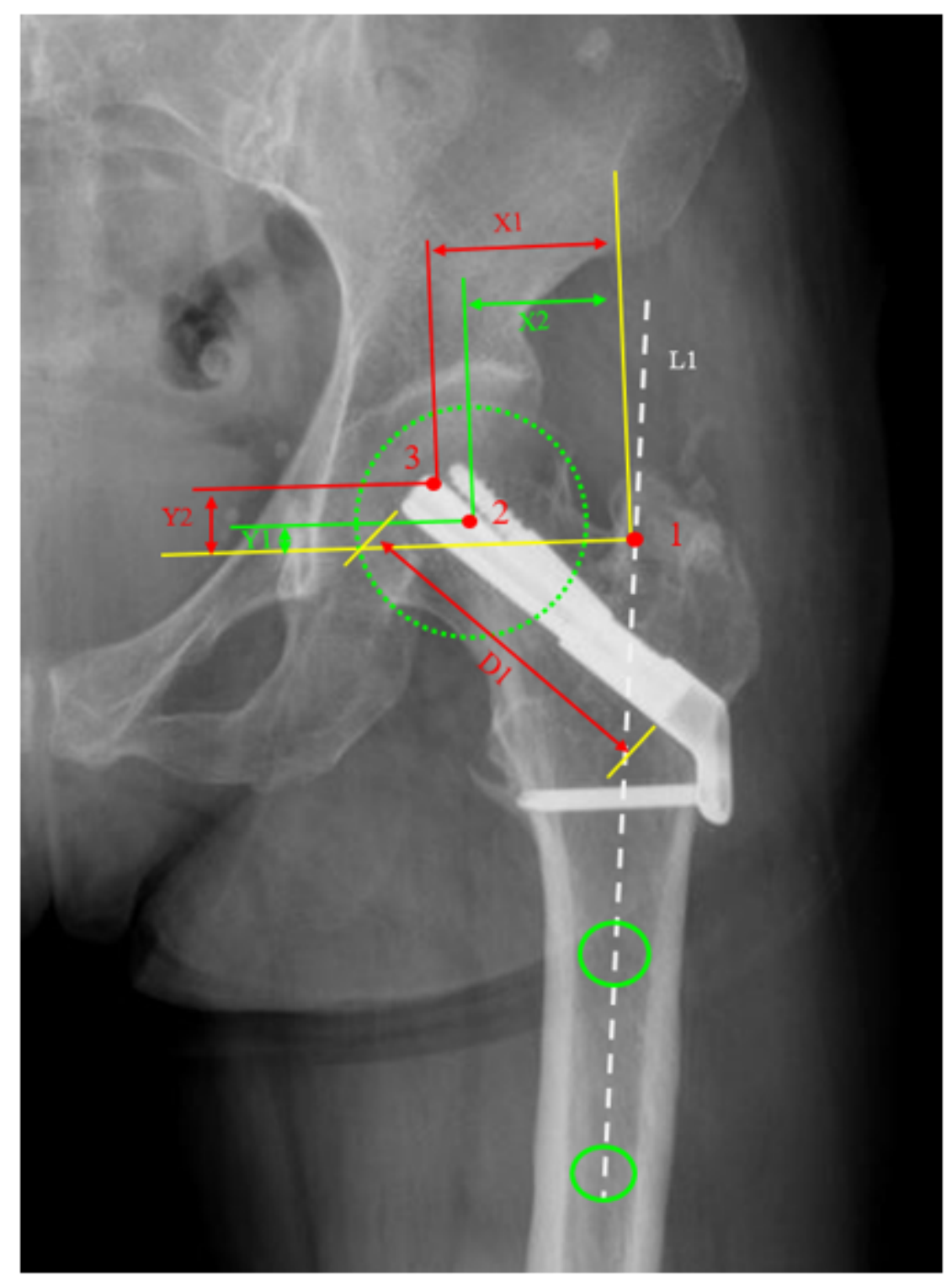

\section{Figure 1}

\section{$\mathrm{XYZ}$-axis measurement.}

1.Determine the femoral shaft axis. Establish two circles in the medullary canal. Draw a straight line(L1) passing the center of both circles until it joins with the piriformis fossa. Then the point of intersection(1) is identified as the static reference point.

2.Determine the center of the femoral head. Establish the best fit circle over femoral head, the center will be the center of the femoral head(2).

3.Determine correction factor for leg rotation. Measure the length of the bolt(D1) and compare with the actual size of the bolt, This ratio(actial size/D1) will be used to correct all measurements in the radiograph. 
4.Determine the $X$ and $Y$ axis based on the point 1,2and 3(point 3 is a virtual point which is determined as the center of the femoral head postoperatively).Measure the linear distance, horizontally $\mathrm{X} 1$ and $\mathrm{X} 2$, vertically $\mathrm{Y} 1$ and $\mathrm{Y} 2$.

5.Caculate the vector sum of DX and DY, then the result is the Femoral neck shortening after correction.
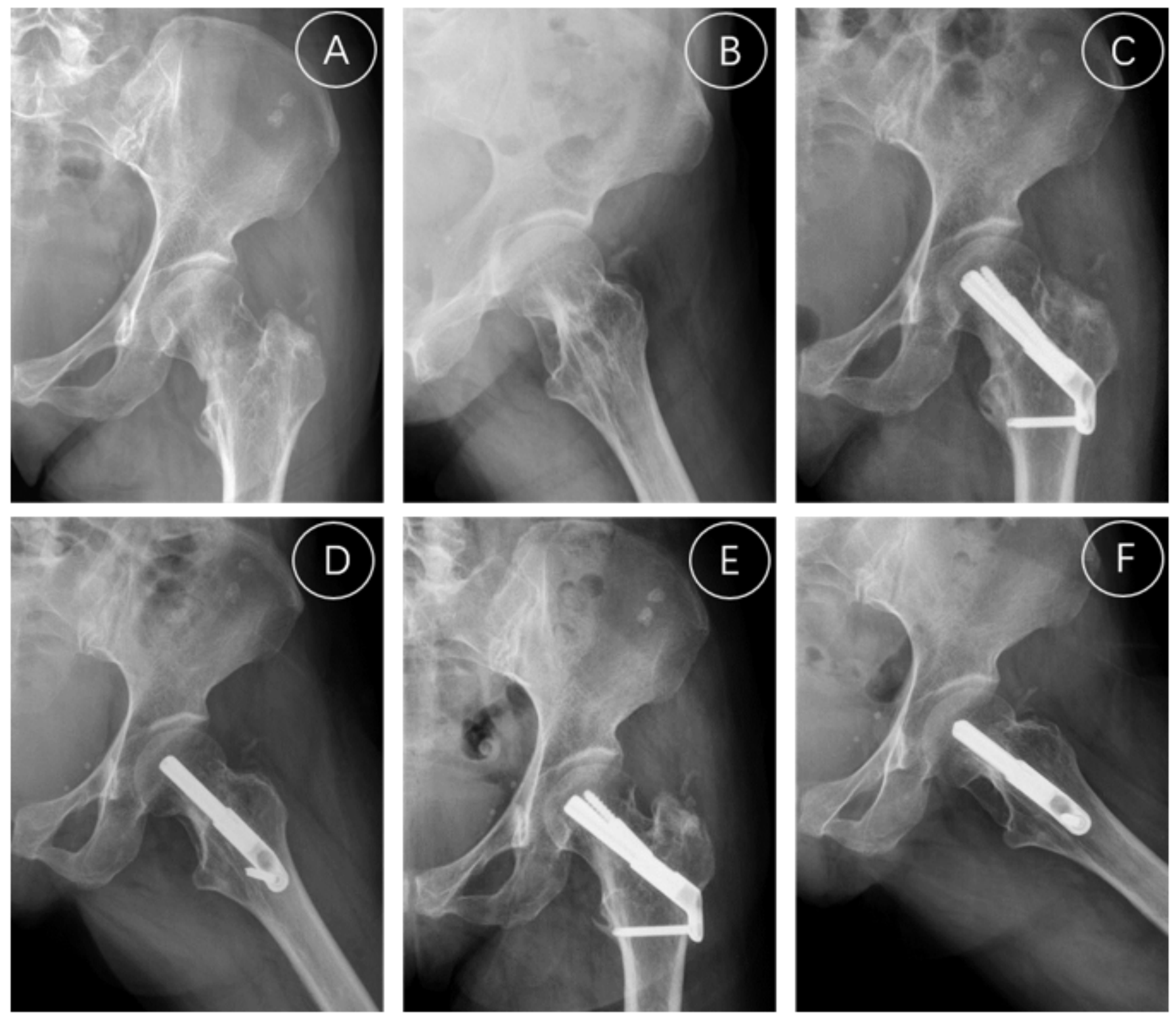

\section{Figure 2}

Typical case: A 53-year-old female fell when she was walking, radiograph showed a left vertical femoral neck fracture(Garden classification type II (A, B)), with a history of ipsilateral intertrochanteric fracture eight years ago, the implant had been removed four years ago. Surgery was performed four days after the injury. On the second day postoperatively, radiography showed good position of FNS and excellent quality of reduction (C, D). Bone healing was achieved on four months postoperatively demonstrated by radiography, then radiography showed no osteonecrosis on one year after surgery $(E, F)$, Harris score of left hip joint: 88 points. 